\title{
Aerodynamics Optimization of a Ducted Coaxial Rotor in Forward Flight Using Orthogonal Test Design
}

\author{
Yuening Jiang $\mathbb{D}^{1,2}$ Hai Li, ${ }^{1,2}$ and Hongguang Jia ${ }^{1,3}$ \\ ${ }^{1}$ Changchun Institute of Optics, Fine Mechanics and Physics, Chinese Academy of Sciences, Changchun 130033, China \\ ${ }^{2}$ University of Chinese Academy of Sciences, Beijing 100049, China \\ ${ }^{3}$ Chang Guang Satellite Technology Co., Ltd., Changchun 130000, China
}

Correspondence should be addressed to Yuening Jiang; atpynjiang@163.com

Received 30 September 2017; Revised 11 January 2018; Accepted 30 January 2018; Published 28 May 2018

Academic Editor: Mickaël Lallart

Copyright (C) 2018 Yuening Jiang et al. This is an open access article distributed under the Creative Commons Attribution License, which permits unrestricted use, distribution, and reproduction in any medium, provided the original work is properly cited.

\begin{abstract}
To investigate the aerodynamic complexities involved in the combination of freestream and propeller's suction flow field of ducted coaxial rotors system in forward flight, an orthogonal $L_{16}\left(4^{3}\right)$ test design has been applied to optimize the design parameters including forward speed, pitch angle, and axial spacing between rotors. Multiblock grids and Multiple Frame of Reference (MFR) method are adopted for calculating aerodynamic performance of the system, hover characteristic was compared with experimental data obtained from the test stand, and the thrust performance is well predicted for various rotor spacing and a range of rpm. This solution approach is developed for the analytical prediction of forward flight and the simulation results indicated that the design parameters influenced lift, drag, and torque reduced in the order: wind speed $>$ rotor spacing $>$ pitch angle, wind speed $>$ pitch angle, and rotor spacing $>$ wind speed $>$ pitch angle, respectively. The optimal rotor spacing and pitch angle were determined to maximize the aerodynamic performance considering high lift, low drag, and trimmed torque.
\end{abstract}

\section{Introduction}

The small unmanned aerial vehicles (SUAVs) with ducted rotor configuration offer a number of significant advantages over other vertical take-off and landing (VTOL) flight vehicles [1]. In recent decades, they generated considerable interests in both military and civilian applications. Practical uses for these flight vehicles have extended to aerial mapping, disaster surveillance, and military reconnaissance [2]. Security is notably improved through shrouding an isolated rotor within a duct, which decreases the possibility of rotors striking person or objects.

The efficiency of aerodynamic configuration is critical for the performance of VTOL vehicles [3]. It has been observed that the hovering rotor system produces more aerodynamic efficiency with duct, which generates higher total thrust due to low pressure region on the duct lip and intensifies inflow distribution by accelerating flow towards the outboard sections of interior rotors $[4,5]$. Since coaxial counterrotating rotors produce the net thrust instead of an isolated rotor design in conventional way, the diameter of the rotors can be decreased to take the same amount of weight $[6,7]$. Compared to single rotor system, the torque generated by coaxial rotor can be opposed by the counterrotating rotor; therefore, no flow deflector or rudder reflection is required.

Unlike the fixed-wing aircraft vehicles or conventional rotational wing vehicles, on the ducted contrarotating rotors, the wake of each rotor is significantly interacted with one another and the tip-vortex is highly influenced by the shrouded duct structure [8-10]. Additionally, for a fixed pitch angle system the various flight velocities produce axial flow through the rotors that increase the angle of attack (AOA) of blade section in the forward flight.

Computational fluid dynamics (CFD) is adopted to analyze complex viscous flow in a wide range of flight conditions. Genç [11] verified the $k-k_{L}-\omega$ transition model which predicted more accurate laminar separation and reattachment of thin airfoil by comparison with $k-\omega$ SST transition model, $k-\omega$ SST turbulence model, and $k-\varepsilon$ turbulence model. Then, Genç et al. [12-14] numerically simulated the elimination of separation bubble in the application of transition models by fluent. Lakshminarayan and Baeder [15] used compressible 
TABLE 1: Forward flight aerodynamics factor-level form.

\begin{tabular}{|c|c|c|c|c|c|c|}
\hline \multirow{2}{*}{$\begin{array}{l}\text { Factor level } \\
1\end{array}$} & \multicolumn{2}{|c|}{ Pitch angle $\left(\alpha /^{\circ}\right)$} & \multicolumn{2}{|c|}{ Flight velocity $\left(v / \mathrm{m}^{\prime} \mathrm{s}^{-1}\right)$} & \multicolumn{2}{|c|}{ Rotor spacing $(\mathrm{H} / \mathrm{mm})$} \\
\hline & $A_{1}$ & 85 & $\mathrm{~B}_{1}$ & 5 & $\mathrm{C}_{1}$ & 38 \\
\hline 2 & $\mathrm{~A}_{2}$ & 80 & $\mathrm{~B}_{2}$ & 10 & $\mathrm{C}_{2}$ & 57 \\
\hline 3 & $\mathrm{~A}_{3}$ & 75 & $\mathrm{~B}_{3}$ & 15 & $\mathrm{C}_{3}$ & 66.5 \\
\hline 4 & $\mathrm{~A}_{4}$ & 70 & $\mathrm{~B}_{4}$ & 20 & $\mathrm{C}_{4}$ & 76 \\
\hline
\end{tabular}

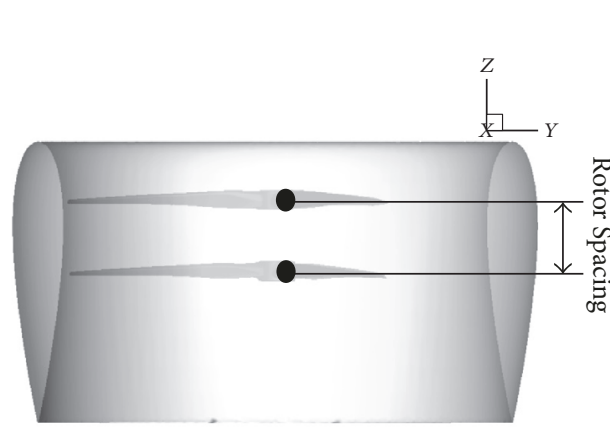

(a) Rotor spacing of the coaxial rotors

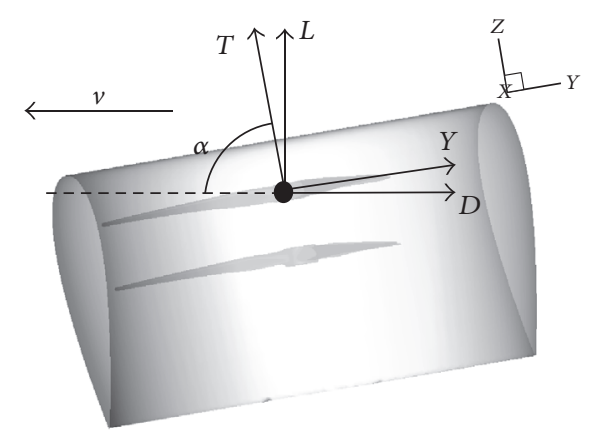

(b) Pitch angle and forward flight velocity

FIGURE 1: Configuration of VTOL aircraft in forward flight.

RANS solver with sliding mesh interpolation scheme to predict instantaneous thrust and power influenced by the interaction between coaxial rotors. Singh et al. [16] used lowand high-fidelity with loose coupling method to computational simulated steady and unsteady performance of elastic coaxial rotor with vibratory hubloads.

Experiment techniques for aircraft vehicles have also developed significantly over time. Schmaus and Chopra [17] evaluated the vibratory loads of rigid coaxial rotor for testing in the wind tunnel and explored the influence of rotor phase on vibratory hubloads. Genç et al. [18] performed an experimental study on the pressure distribution and aerodynamic forces of NACA2415 aerofoil at low Reynolds carried out in the suction-type wind tunnel.

Many experimental and numerical efforts had been done to investigate the forward flight performance [1923]. However, most previous studies were concentrated on optimizing the shape of duct or measuring the aerodynamic step-by-step. Rarely has research been proposed to maximize the fundamental aerodynamic performance issues, which are associated with the determination of the rotor spacing between upper and lower rotors, taking into account both attitude and speed of the ducted coaxial rotors configuration.

In this work, the identical three-dimensional ducted propeller configuration has been established to validate the efficiency of numerical scheme in achieving high-fidelity predictions of hybrid flow field and compared with the experiments measured by the hovering aerodynamic performance test stand of our laboratory. The aim of the hover performance investigation is to apply this analytical approach to forward flight. Besides, this solving method aims to form the fundamental exploring correlation between vibratory loads and blade loads, thereby aiding in a better selection of blade structural properties in manufacturing. Orthogonal test design is designed to analyze the dominant factor to each aerodynamics. Subsequently, the variation trends of aerodynamic performance are characterized with three leading influence factors including pitch angle, freestream velocity, and rotor spacing. The optimized rotor spacing and flight pitch angle are determined from a wide range of factors to maximize forward flight performance of our system.

\section{Orthogonal Test Design}

According to experimental purpose, three influence factors, given by incident angle to the freestream in forward flight $\left(\alpha /{ }^{\circ}\right)$, flight speed $\left(v / \mathrm{m} \cdot \mathrm{s}^{-1}\right)$ and rotor spacing $(\mathrm{H} / \mathrm{mm})$ are chosen and the level of factors have been decided. Variation tendency of aerodynamic performance with proposed four factor levels for each influenced factor can be compared accurately and optimum parameter combination can be designed with three factors taken into consideration. Corresponding to the four levels of pitch angles listed in Table 1, wind speed is given by $5 \mathrm{~m} / \mathrm{s}, 10 \mathrm{~m} / \mathrm{s}, 15 \mathrm{~m} / \mathrm{s}$, and $20 \mathrm{~m} / \mathrm{s}$ and rotor spacing represents the distance between top and bottom rotor ranging from $38 \mathrm{~mm}$ to $76 \mathrm{~mm}$. Figure 1(a) shows the longitudinal forces acting on the calculation configuration in forward flight; then lift and drag can be calculated as follows:

$$
\begin{aligned}
& L=T \sin \alpha+Y \cos \alpha, \\
& D=Y \sin \alpha-T \cos \alpha,
\end{aligned}
$$

where $T$ and $Y$ refer to thrust and tangential force, which can be predicted by numerical approach.

Orthogonal array $L_{16}\left(4^{3}\right)$ is adopted to optimize the prescription of forward flight ducted coaxial rotors [24]. Table 2 summarizes the numerical calculation scheme. 
TABLE 2: Test number of forward flight aerodynamics.

\begin{tabular}{lccc}
\hline Test number & $\alpha /^{\circ}$ & $v / \mathrm{m}^{-1}$ & $\mathrm{H} / \mathrm{mm}$ \\
\hline 1 & $\mathrm{~A}_{1}$ & $\mathrm{~B}_{1}$ & $\mathrm{C}_{1}$ \\
2 & $\mathrm{~A}_{1}$ & $\mathrm{~B}_{2}$ & $\mathrm{C}_{2}$ \\
3 & $\mathrm{~A}_{1}$ & $\mathrm{~B}_{3}$ & $\mathrm{C}_{3}$ \\
4 & $\mathrm{~A}_{1}$ & $\mathrm{~B}_{4}$ & $\mathrm{C}_{4}$ \\
5 & $\mathrm{~A}_{2}$ & $\mathrm{~B}_{1}$ & $\mathrm{C}_{2}$ \\
6 & $\mathrm{~A}_{2}$ & $\mathrm{~B}_{2}$ & $\mathrm{C}_{3}$ \\
7 & $\mathrm{~A}_{2}$ & $\mathrm{~B}_{3}$ & $\mathrm{C}_{4}$ \\
8 & $\mathrm{~A}_{2}$ & $\mathrm{~B}_{4}$ & $\mathrm{C}_{1}$ \\
9 & $\mathrm{~A}_{3}$ & $\mathrm{~B}_{1}$ & $\mathrm{C}_{3}$ \\
10 & $\mathrm{~A}_{3}$ & $\mathrm{~B}_{2}$ & $\mathrm{C}_{4}$ \\
11 & $\mathrm{~A}_{3}$ & $\mathrm{~B}_{3}$ & $\mathrm{C}_{1}$ \\
12 & $\mathrm{~A}_{3}$ & $\mathrm{~B}_{4}$ & $\mathrm{C}_{2}$ \\
13 & $\mathrm{~A}_{4}$ & $\mathrm{~B}_{1}$ & $\mathrm{C}_{4}$ \\
14 & $\mathrm{~A}_{4}$ & $\mathrm{~B}_{2}$ & $\mathrm{C}_{1}$ \\
15 & $\mathrm{~A}_{4}$ & $\mathrm{~B}_{3}$ & $\mathrm{C}_{2}$ \\
16 & $\mathrm{~A}_{4}$ & $\mathrm{~B}_{4}$ & $\mathrm{C}_{3}$ \\
\hline
\end{tabular}

\section{Numerical Simulation}

3.1. Solving Method. The flow field is divided into multisubdomains to solve the complexity of suction flow and frontflow in forward flight. Interface boundary condition has been established between subdomains to keep flux conservation coupled with unstructured overset mesh. Figure 2 shows the computational rotor. Spatial discretization of inviscid terms is computing using Roe's flux difference splitting format, and second-order central differencing is employed to compute viscous terms. Time integration is discrete using second-order implicit difference scheme. Spalart-Allmaras turbulence model is employed to account for the turbulence in the vortex core attributed to the rotating effect.

Multiple frames of reference (MFR) have been used to analyze the situation involving domains that are rotating relative to stationary domains that allow for the adequate predictions of complex flows dominated by transient interactions of the relative movement between rotating and stationary domains.

Moving coordinate system rotates at the angular velocity $\vec{\omega}$ relatively to the stationary coordinate system, the unit vector $\vec{a}$ represents rotating axis as seen in Figure 3 . The origin of rotation coordinate system is defined by the vector $\overrightarrow{r_{0}}$ associated with stationary system. Thus $\vec{\omega}$ can be described as

$$
\vec{\omega}=\omega \vec{a}
$$

Any certain point in the computational domain of rotation system is determined by position vector $\vec{r}$ and the origin point. The transformation of velocity from static system to rotated system can be realized as follows:

$$
\vec{v}_{r}=\vec{v}-\vec{u}_{r}
$$

In (3), $\vec{v}_{r}$ and $\vec{v}$ represent the relative velocity and the absolute velocity, respectively. $\vec{u}_{r}=\vec{\omega} \times \vec{r}$ is the converted acceleration.

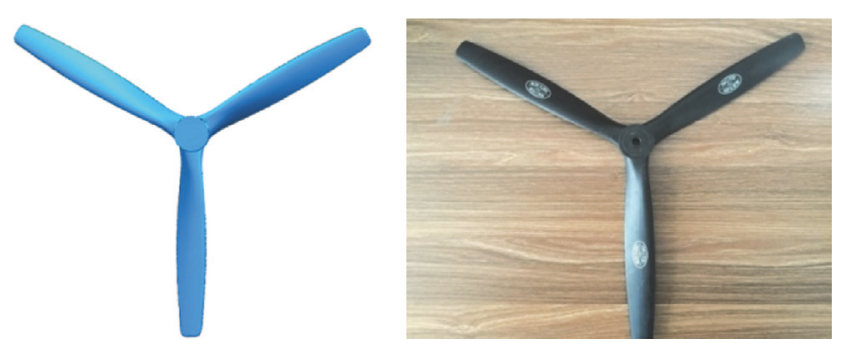

Figure 2: Computational model of rotor.

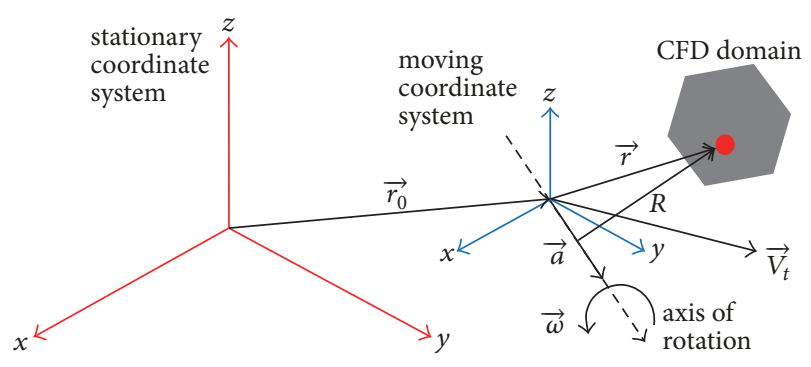

FIGURE 3: Sketch of multiframe reference model.

Additional terms in momentum equation lead to the increase of fluid acceleration, derived from the multiple motion individual problems. As a consequence, governing equations can be expressed in two ways. The momentum equation, which is produced on the basis of relative and absolute velocity separately, forms the foundation of fluid governing equations. The governing equations of fluid mechanics in terms of relative velocity can be written as follows:

Continuity Equation

$$
\frac{\partial \rho}{\partial t}+\nabla \cdot \rho \vec{v}_{r}=0
$$

Momentum Equation

$$
\begin{aligned}
& \frac{\partial}{\partial t}\left(\rho \vec{v}_{r}\right)+\nabla \cdot\left(\rho \vec{v}_{r} \vec{v}_{r}\right) \\
& \quad+\rho\left(2 \vec{\omega} \times \vec{v}_{r}+\vec{\omega} \times \vec{\omega} \times \vec{r}\right) \\
& =-\nabla p+\nabla \cdot \vec{\tau}+\vec{F} .
\end{aligned}
$$

In (5), Coriolis acceleration term $2 \vec{\omega} \times \vec{v}_{r}$ and centripetal acceleration term $\vec{\omega} \times \vec{\omega} \times \vec{r}$ are two additional kinds of acceleration.

Energy Equation

$$
\frac{\partial}{\partial t}\left(\rho E_{r}\right)+\nabla \cdot\left(\rho \vec{v}_{r} H_{r}\right)=\nabla \cdot\left(k \nabla T+\overline{\overline{\tau_{r}}} \cdot v_{r}\right)+s_{h}
$$

where $\overline{\overline{\tau_{r}}}$ represents the viscous stress term in the form of relative velocity derivation. In (6), $E_{r}=h-p / \rho+(1 / 2)\left(v_{r}^{2}-u_{r}^{2}\right)$ is defined to be the relative intrinsic energy and $H_{r}=E_{r}+p / \rho$ is relative total enthalpy. 
The controlling equations with respect to absolute velocity format in rotation coordinate system can be reevaluated as (7).

$$
\begin{aligned}
& \frac{\partial \rho}{\partial t}+\nabla \cdot \rho \vec{v}_{r}=0, \\
& \frac{\partial}{\partial t} \rho \vec{v}+\nabla \cdot\left(\rho \vec{v}_{r} \vec{v}\right)+\rho(\vec{\omega} \times \vec{v}) \\
& \quad=-\nabla p+\nabla \cdot \vec{\tau}+\vec{F}, \\
& \frac{\partial}{\partial t} \rho E+\nabla \cdot\left(\rho \vec{v}_{r} H+p \vec{u}_{r}\right)=\nabla \cdot(k \nabla T+\overline{\bar{\tau}} \cdot v)+s_{h} .
\end{aligned}
$$

The Coriolis acceleration and centripetal acceleration are attributed to the individual $\vec{\omega} \times \vec{v}$ term.

3.2. Boundary Conditions and Mesh Generation. A cylindrical flow field has been established to surround the ducted rotors; velocity inlet and pressure outlet boundary conditions were specified on the far-field planes, as shown in Figure 4. The diameter and height of the column are $4 D$ and $7 D$, where $D$ represents the duct outer diameter and the calculation model is placed at $4 D$ from inflow boundary. The coaxial rotor is based on the NACA airfoil and it is paired with duct based on the NACA 0012 airfoil. Shrouding the double rotors configurations within the duct can be seen in Figure 5. Unstructured overset grids have been used to allow for grid densification at the blade tip clearance in achieving high-fidelity simulation of leakage vortex. Nodes were also clustered at the duct lip and near the diffusional section of duct.

In this current study, $2.5,3.5,4.5$, and 5.5 million numbers of grid nodes have been used to discuss the independence of computing grid, which maintain a marginal variation in solutions at a stage of grid quantity [12]. Hovering thrust as well as the trimmed torque of two counterrotating rotors for entire system is obtained by MFR model for all the grid sizes in Table 3 and compares with the experimental data. Typical convergence trend of torque is readily discovered; the number of grid has been increased by 1 million as the torque reduces to within $1 \%$ with 5.5 million grids, when the variation of thrust is less than $2 \%$ approximately. The size of grid is determined after grid independence test and the computing results are in accord with experiments well. Spatial slice mesh of interior rotational domains and exterior stationary domains around coaxial rotors are shown in Figure 6 with adequate mesh accuracy and resolution.

\section{Experimental Investigation for Hover Aerodynamic Performance}

The hover efficiency affected by RPM and rotor spacing are critical for the implement of duct coaxial rotor UAV [25, 26]. In this section, a hover test stands for ducted coaxial rotor model which was established in order to experimentally measure the performance in hover, as shown in Figure 7. The identical 3-D geometries were tested to assess the effect of multiblock generation and MFR solve method by comparing

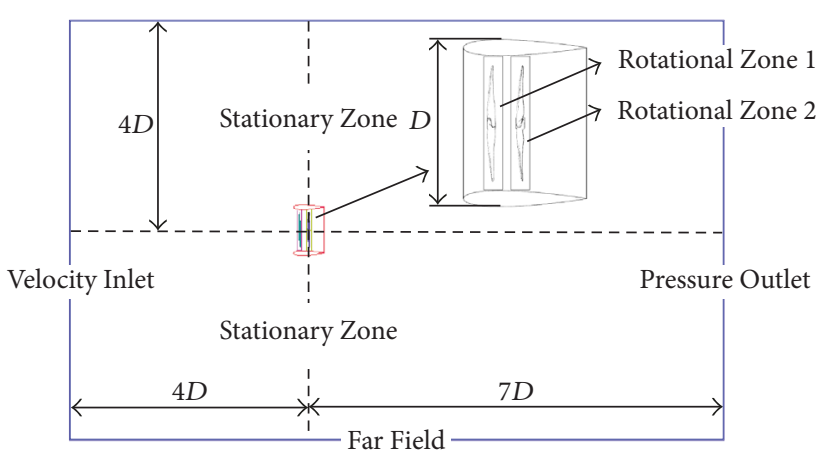

FIgURE 4: Computational domains of ducted coaxial rotors.

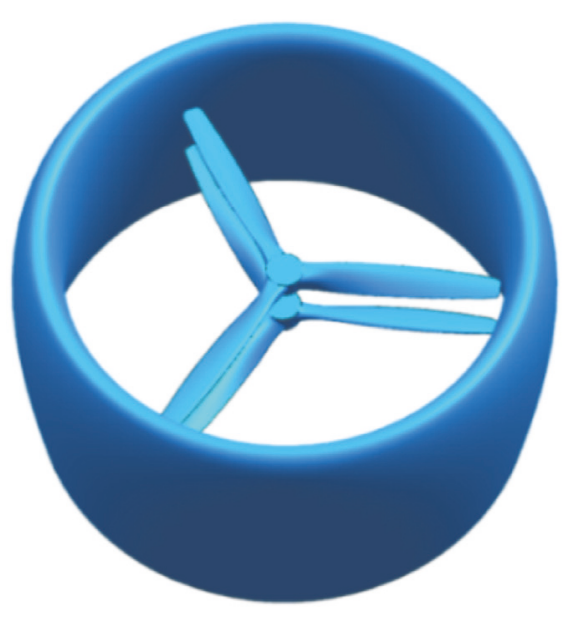

FIGURE 5: Geometric model of ducted coaxial rotor.

it with the experimental results. The accuracy and efficiency of current numerical simulation indicated that the application of our method is capable of continuing to be utilized for forward aerodynamic computation in the next section.

The rotor and motor systems were mounted inversely on hinges. Two physical quantities can be simultaneously measured by designed test stand. (1) Thrust: thrust was measured by the weighing sensor, which was placed between the inner frame structure and external frame structure. (2) Rotational speed: considering the free rotation and independent transmission, the rotational speed of each rotor was separately determined through an approach switch that was sensed by metal objects in the range of $5 \mathrm{~mm}$.

Simulation of total thrust produced by the entire ducted coaxial rotor system is validated by the experimental data in Figure 8. A wide range of rpm varying from 1000 to 7000 were conducted to maximize the aerodynamic capability of testing model. The calculated thrusts agreed well with the measurement data at low speed, whereas at higher speed the analytical result is slightly underpredicted for the given thrust level and the disparities increased as the rotational speed raised. The computation assigning identical rotational speed for the top and bottom rotors that generated thrusts in the same trends with variation of rpm, while the upper rotor is more efficiency than lower one, which is affected by 
TABLE 3: Hovering thrust and trimmed torque versus mesh quantity based on MFR model at $6000 \mathrm{rpm}$.

\begin{tabular}{|c|c|c|c|c|c|}
\hline $6000 \mathrm{rpm}$ & $2.5 \times 10^{6}$ & $3.5 \times 10^{6}$ & $4.5 \times 10^{6}$ & $5.5 \times 10^{6}$ & Expt. \\
\hline$T(\mathrm{~kg})$ & 30.588 & 32.682 & 33.991 & 34.636 & 37.318 \\
\hline$Q(\mathrm{~N} \cdot \mathrm{m})$ & 0.111 & 0.108 & 0.106 & 0.105 & 0.103 \\
\hline
\end{tabular}
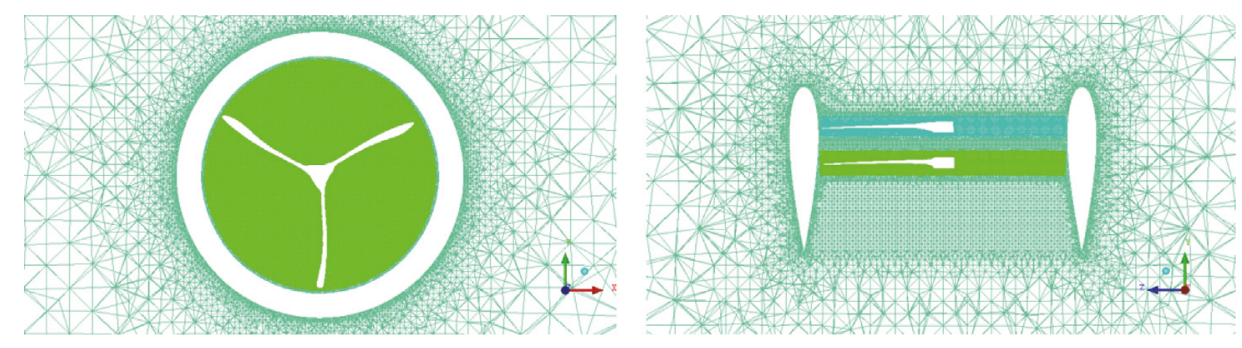

FIGURE 6: Space grids slices of nested grid system (grid quantity: $5.5 \times 10^{6}$ ).

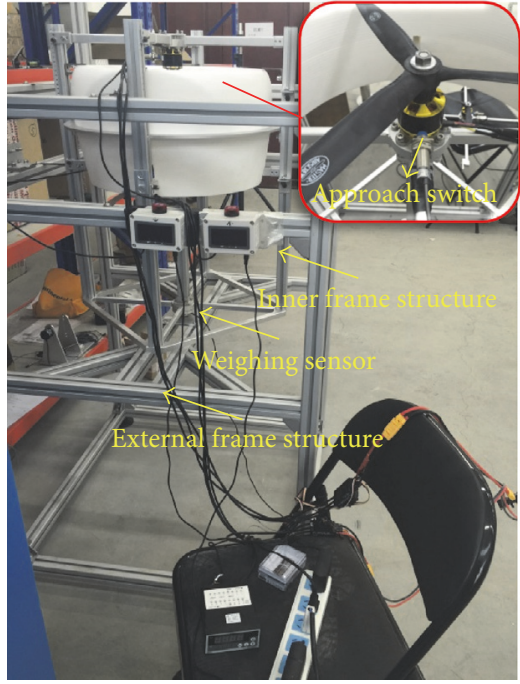

FIGURE 7: Structural components of hover test stand.

the contracted slipstream of upper one. The thrust of duct structure is seen to remain fairly equal to the lower rotor.

Figure 9 shows the total thrust of the entire system obtained from the test stand experiment for a range of distance between rotors varying from 3000 to 7000 . The rotor spacing is given by $H / R=0.25,0.35,0.45,0.55$, and 0.65 . In hover performance, the effect of rotor spacing is not significant for the thrust, which approaches a constant value ranged from 0.25 to 0.4 , while the thrust abruptly decreased at 0.45 .

\section{Results and Discussion}

Since a variety of physical parameters influence the forward performance, the optimization of the structure form of essential aerodynamic component and the aerodynamic characteristic is a critical step in the development of a SUAV. The angle of attack of duct, freestream velocity, and rotor spacing of the coaxial rotors are generally considered to be

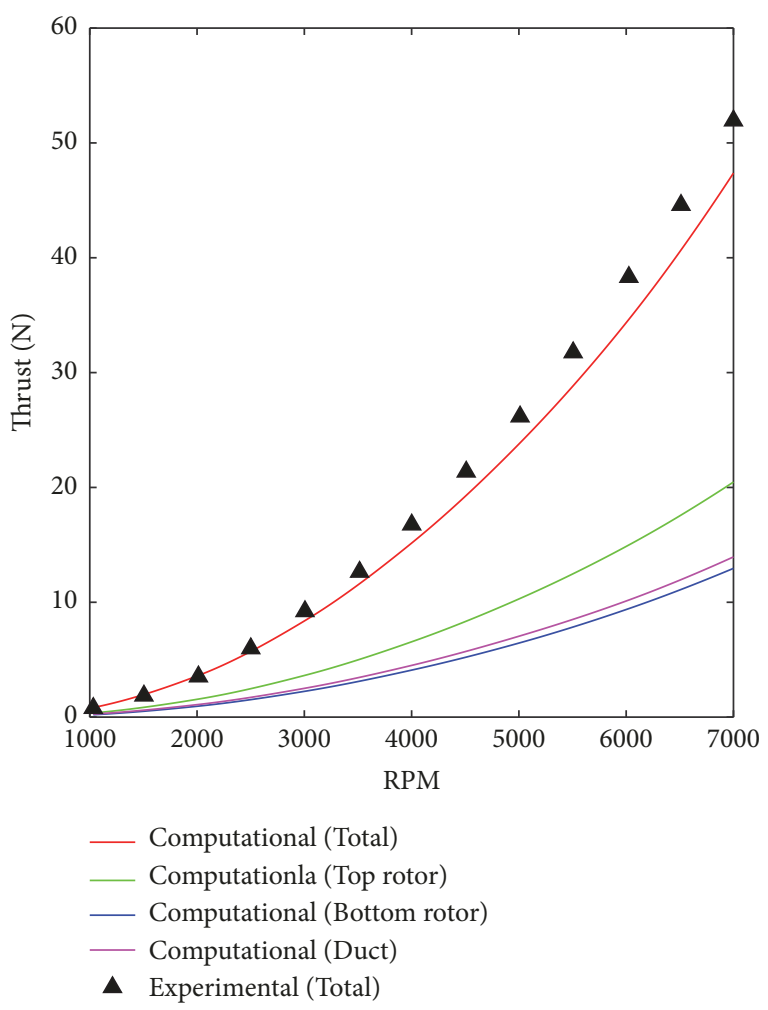

FIGURE 8: Hover performance comparison with experimental data at different rpm.

the most crucial factors. The procedure traditionally used for optimization the structural configuration of ducted coaxial rotors can be implemented by experimental investigation or carried out step-by-step. In the current research, all selected factors were tested using an orthogonal $L_{16}\left(4^{3}\right)$ test design.

Results of each scheme are summarized in Table 4; the calculation scheme $\mathrm{A} 1 \mathrm{~B} 4 \mathrm{C} 4$ provided the maximum lift $(49.82 \mathrm{~N})$. The minimum drag of total system and the minimum torque value trimmed of counterrotating coaxial rotor were $0.4707 \mathrm{~N}(\mathrm{~A} 2 \mathrm{~B} 1 \mathrm{C} 2)$ and $0.0178 \mathrm{~N} \cdot \mathrm{m}(\mathrm{A} 4 \mathrm{~B} 2 \mathrm{C} 1)$, 


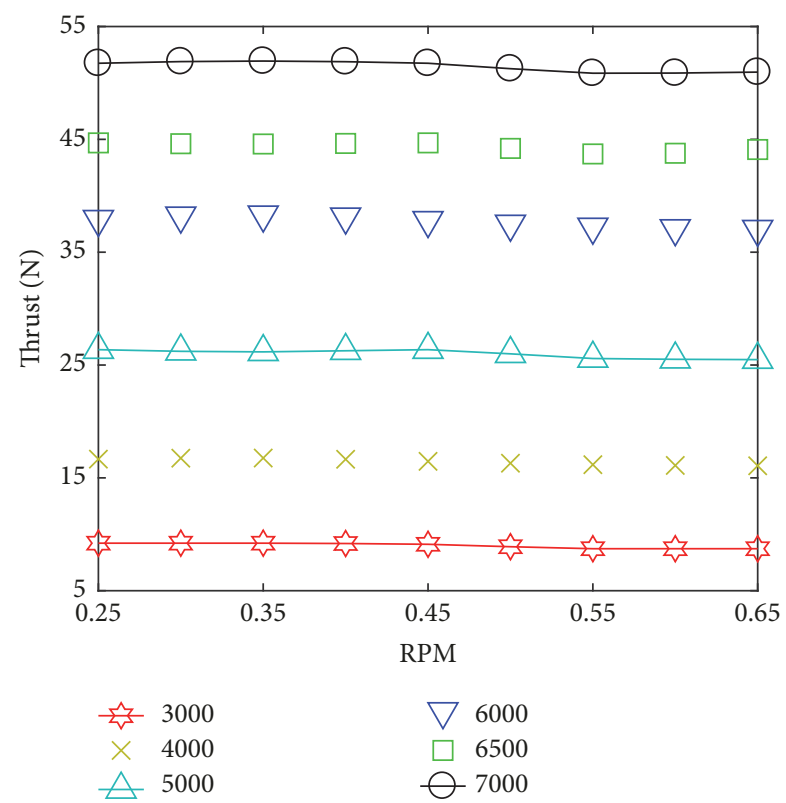

FIGURE 9: Experimental thrust versus rotor spacing.

TABLE 4: $L_{16}\left(4^{3}\right)$ test results of aerodynamics.

\begin{tabular}{lccc}
\hline Test number & Lift/N & Drag/N & Torque/ N·m \\
\hline 1 & 24.3606 & 2.6550 & -0.0527 \\
2 & 35.9317 & 13.2894 & 0.1087 \\
3 & 42.2899 & 26.3460 & 0.2102 \\
4 & 49.8214 & 41.1217 & 0.1928 \\
5 & 33.1124 & 0.4707 & 0.1139 \\
6 & 37.2669 & 9.3868 & 0.1472 \\
7 & 43.7340 & 22.2020 & 0.1885 \\
8 & 42.1831 & 39.3675 & 0.1514 \\
9 & 33.8219 & -1.8496 & 0.1246 \\
10 & 36.8556 & 6.3430 & 0.1714 \\
11 & 35.0175 & 24.7934 & 0.0773 \\
12 & 43.4715 & 34.1595 & 0.2029 \\
13 & 33.3692 & -4.5041 & 0.1366 \\
14 & 27.1594 & 7.6410 & -0.0178 \\
15 & 40.3194 & 16.2937 & 0.1446 \\
16 & 48.0299 & 31.6043 & 0.2060 \\
\hline
\end{tabular}

respectively. By now, we cannot determine the best combination of levels depending on these consequences simply. Furthermore, an orthogonal analysis has been exhibited in terms of the test results of aerodynamics in Table 4. The statistical model including the sum of aerodynamic force $T$, mean value $m$, and range value was computed to analysis $L_{16}\left(4^{3}\right)$ calculation results in Table 5.

According to the range value of factors, the influence to the lift, drag, and torque reduced in the order $\mathrm{B}>\mathrm{C}>\mathrm{A}$, $\mathrm{B}>\mathrm{A}>\mathrm{C}$, and $\mathrm{C}>\mathrm{B}>\mathrm{A}$, respectively. Wind speed of freestream was demonstrated to be the most significant determination of lift and drag and the value of trimmed torque is most decided by the spacing of rotors.
Figure 10 compares the mean values of lift, drag, and torque $\left(m_{i}\right)$ analytically calculated in Table 5 versus four factor levels. Lift and torque show the same trends with rotor spacing, which almost have no effect on drag force. Wide rotor spacing is one of the main contributors to lift force that is capable of decreasing the interference between upper and lower rotors. In order to optimize lift of system, $76 \mathrm{~mm}$ should be adopted for the spacing of coaxial rotor. Thrust remains fairly constant value with rotor spacing in hover. While in forward flight, the thrust sharply increases with rotor spacing due to flow asymmetry in freestream.

Air volume intake into duct system correspondingly increases with freestream velocity as per unit time. Differential pressure between the upper and lower area of propeller is enlarged attribute to the more remarkable suction flow. As a consequence, wind speed is found to be the most determinant factor of lift in Figure 10(a). Meanwhile, drag is highly magnifying due to inhibition effect of duct as the flight speed increasing, which can be seen in Figure 10(b). To reduce the drag of forward flight, a small amount of wind speed is the most significant effect factor.

Pitch angle of ducted coaxial rotor has no significant influence on the aerodynamic force. In the meantime, angle of attack marginally affects drag force that the increase of deflection away from edgewise forward direction (pitch angle at $90^{\circ}$ ) would lead to small amount of drag reduction. That is because, at lower pitch angle, a slight decrease of negative pressure zone occurs near internal lip of duct at wind side and the drag reduces with the pressure differential between external and internal side. Furthermore, at upwind side, the lessening of high pressure zone near internal lip at upwind side also contributes to the drag reduction. In forward flight, for fixed rpm assuming in this calculations, $70^{\circ}$ is more suitable for the ducted coaxial rotor system to achieve minimum power consumption on drag that contributes to better aerodynamic performance.

\section{Conclusions}

For the rotary-wing aircraft vehicles, various structure parameters and flight conditions affect the inflow distribution, making it very challenging to draw a general conclusion about how to practically analyze the aerodynamic characteristic and obtain the optimum performance.

A representative ducted coaxial rotor configuration proposed in this work is motivated by the development of a VTOL aircraft. In this present study MFR model is adopted to predict the complex viscous flow of multirotors, which transform the time-dependent formulation into steady state approximation. The predictive capability of the new configuration as well as the SUAV is very high and validated by the experimental results.

In forward flight, pitch angle, freestream velocity, and rotor spacing between the counterrotating rotors are proved to be three dominant influence factors of aerodynamic performance in previous efforts. Three factors should be taken into consideration in order to optimize lift, drag, and torque of the system. The orthogonal $L_{16}\left(4^{3}\right)$ test design was applied to select the optimum structure and forward 


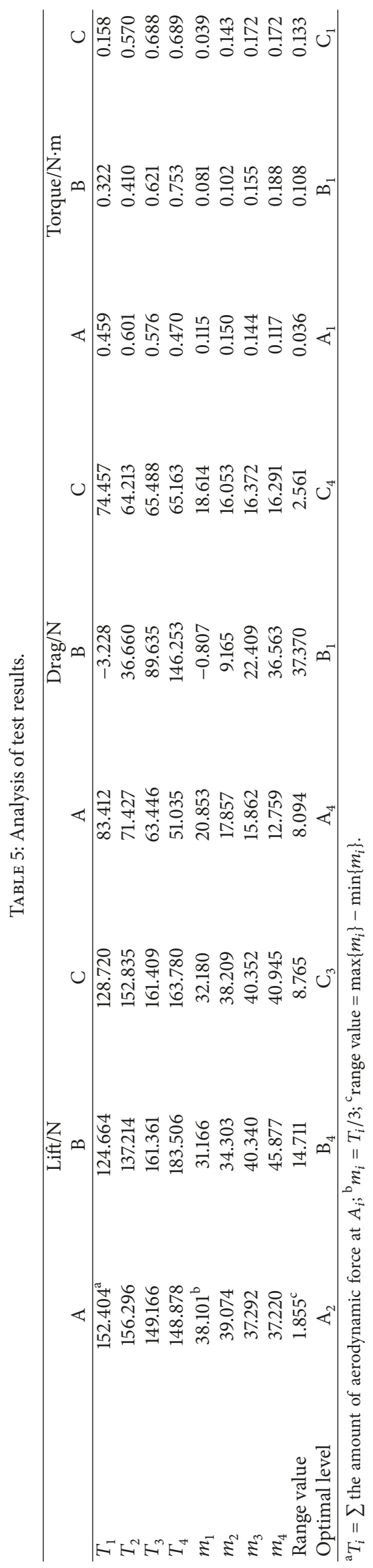




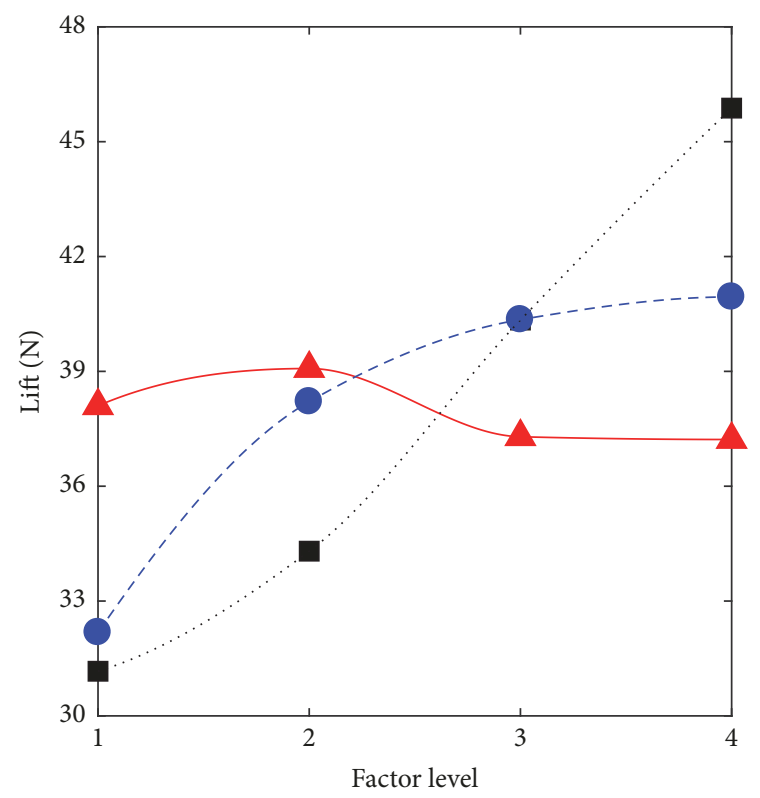

A Pitch angle

Freestream velocity

Rotor spacing

(a) Lift versus level factor

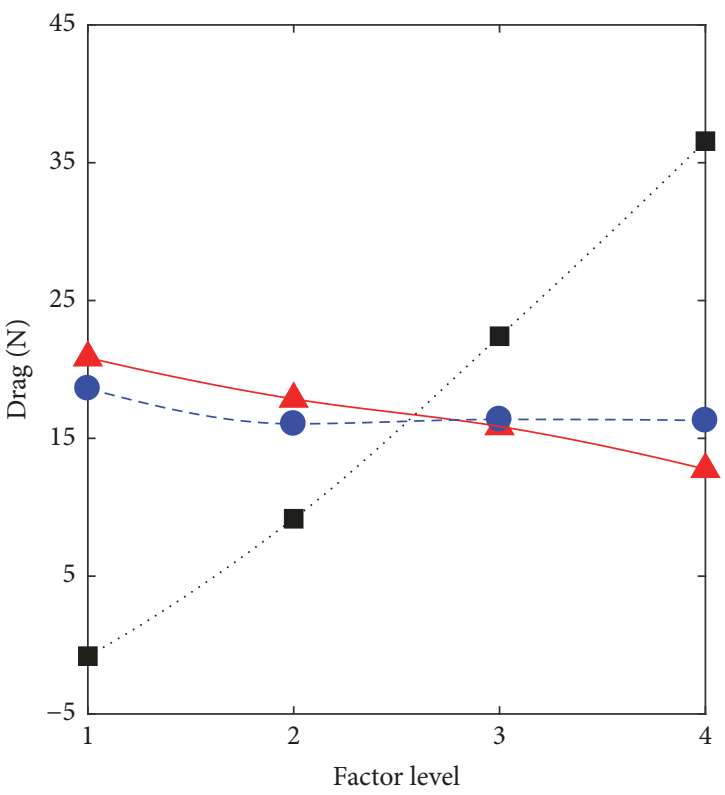

A Pitch angle

Freestream velocity

Rotor spacing

(b) Drag versus level factor

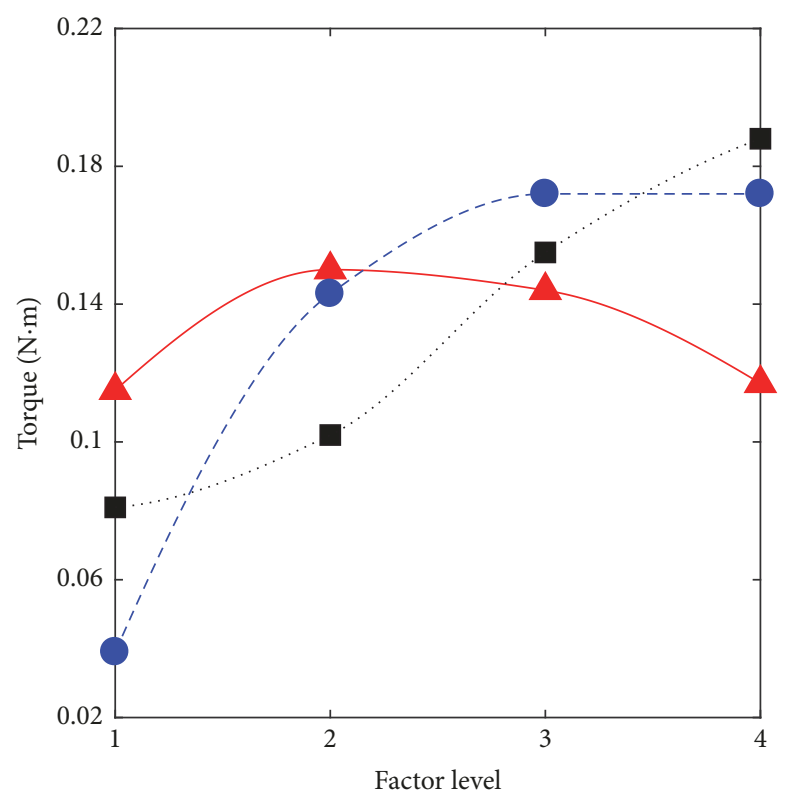

Pitch angle
Freestream velocity
Rotor spacing

(c) Torque versus level factor

FIGURE 10: Trend of forward flight aerodynamics with diverse factors.

flight parameters. Mean values of lift, drag, and torque with four levels of pitch angle, freestream speed, and rotor spacing have been compared and variation trend of the three influence factors has been depicted. Optimal rotor spacing and appropriate forward flight AOA have been decided. The flight speed should be determined by a compromise of high lift and low drag. This study provides an efficiency approach to simulate the mixed flow field of multirotating domains and static domain, which gives out significant reference in engineering application. 


\section{Conflicts of Interest}

The authors declare that they have no conflicts of interest.

\section{Acknowledgments}

This work was supported by the Knowledge Innovation Program of the Chinese Academy of Sciences (Grant no. YYYJ-1122) and the Innovation Program of UAV funded by the Chang Guang Satellite Technology Co., Ltd.

\section{References}

[1] T. E. Lee, Design and performance of a ducted coaxial rotor in hover and forward [Master, thesis], Department of Aerospace Engineering, University of Maryland, College Park, MD, USA, 2010.

[2] F. Bohorquez, Rotor hover performance and system design of an efficient coaxial rotary wing micro air vehicle [Ph.D. thesis], Department of Aerospace Engineering, University of Maryland, College Park, MD, USA, 2007.

[3] V. K. Lakshminarayan and J. D. Baeder, "Computational investigation of microscale coaxial-rotor aerodynamics in hover," Journal of Aircraft, vol. 47, no. 3, pp. 940-955, 2010.

[4] B. G. Jimenez and R. Singh, "Effect of duct-rotor aerodynamic interactions on blade design for hover and axial flight," in Proceedings of the 53rd AIAA Aerospace Sciences Meeting, 2015, USA, January 2015.

[5] J. L. Pereira, Hover and wind-tunnel testing of shrouded rotors for improved micro air vehicle design [Ph.D. thesis], University of Maryland, College Park, MD, USA, 2008.

[6] X. M. He, H. C. Zhao, X. D. Chen, Z. L. Luo, and Y. N. Miao, "Hydrodynamic performance analysis of the ducted propeller based on the combination of multi-block hybrid mesh and Reynolds stress model," Journal of flow control, measurement \& visualization, vol. 3, pp. 67-74, 2015.

[7] O. Rand and V. Khromov, "Aerodynamic optimization of coaxial rotor in hover and axial flight," in Proceedings of the 27th International Congress of the Aeronautical Sciences, Nice, France, 2012.

[8] H. W. Kim and R. E. Brown, "Modelling the aerodynamics of coaxial helicopters - from an isolated rotor to a complete aircraft," in Proceedings of the 1st EU-Korea Conference on Science and Technology, EKC 2008, pp. 45-59, August 2008.

[9] M. Ramasamy, "Hover performance measurements toward understanding aerodynamic interference in coaxial, tandem, and tilt rotors," Journal of the American Helicopter Society, vol. 60, no. 3, Article ID 032005, 2015.

[10] H. W. Kim and R. E. Brown, "Coaxial rotor performance and wake dynamics in steady and maneuvering flight," in Proceedings of the American Helicopter Society 62nd Annual Forum Proceedings, Phoenix, 2016.

[11] M. S. Genç, "Numerical simulation of flow over a thin aerofoil at a high Reynolds number using a transition model," Proceedings of the Institution of Mechanical Engineers, Part C: Journal of Mechanical Engineering Science, vol. 224, no. 10, pp. 2155-2164, 2010.

[12] M. S. Genç, Ü. Kaynak, and H. Yapici, "Performance of transition model for predicting low Re aerofoil flows without/with single and simultaneous blowing and suction," European Journal of Mechanics - B/Fluids, vol. 30, no. 2, pp. 218-235, 2011.
[13] M. S. Genç, Ü. Kaynak, and G. D. Lock, "Flow over an aerofoil without and with a leading-edge slat at a transitional Reynolds number," Proceedings of the Institution of Mechanical Engineers, Part G: Journal of Aerospace Engineering, vol. 223, no. 3, pp. 217231, 2009.

[14] M. Genc, G. Lock, and U. Kaynak, "An Experimental and Computational Study of Low Re Number Transitional Flows over an Aerofoil with Leading Edge Slat," in Proceedings of the The 26th Congress of ICAS and 8th AIAA ATIO, Anchorage, Alaska.

[15] V. K. Lakshminarayan and J. D. Baeder, "High-resolution computational investigation of trimmed coaxial rotor aerodynamics in hover," Journal of the American Helicopter Society, vol. 54, no. 4, Article ID 042008, 2009.

[16] R. Singh, H. Kang, M. Bhagwat, C. Cameron, and J. Sirohi, "Computational and experimental study of coaxial rotor steady and vibratory loads," in Proceedings of the 54th AIAA Aerospace Sciences Meeting, AIAA Paper, January 2016.

[17] J. H. Schmaus and I. Chopra, "Aeromechanics of rigid coaxial rotor models for wind-tunnel testing," Journal of Aircraft, vol. 54, no. 4, pp. 1486-1497, 2017.

[18] M. S. Genç, I. Karasu, and H. Hakan Açikel, "An experimental study on aerodynamics of NACA2415 aerofoil at low Re numbers," Experimental Thermal and Fluid Science, vol. 39, pp. 252264, 2012.

[19] H. Xu and Z. Ye, "Numerical simulation of unsteady flow around forward flight helicopter with coaxial rotors," Chinese Journal of Aeronautics, vol. 24, no. 1, pp. 1-7, 2011.

[20] C. P. Coleman, "A survey of theoretical and experimental coaxial rotor aerodynamic research," NASA TP-3675, 1997.

[21] J. Russell, L. Sankar, C. Tung, J. Russell, L. Sankar, and C. Tung, "High accuracy studies of the tip vortex structure from a hovering rotor," in Proceedings of the 28th Fluid Dynamics Conference, Snowmass Village,CO,U.S.A..

[22] R. E. Brown and A. J. Line, "Efficient high-resolution wake modeling using the vorticity transport equation," AIAA Journal, vol. 43, no. 7, pp. 1434-1443, 2005.

[23] S. Sunada, K. Tanaka, and K. Kawashima, "Maximization of thrust-torque ratio of a coaxial rotor," Journal of Aircraft, vol. 42, no. 2, pp. 570-572, 2005.

[24] Y. Lei, Y. Bai, and Z. Xu, "Wind Effect on Aerodynamic Optimization for Non-planar Rotor Pairs using Full-scale Measurements," Journal of Intelligent \& Robotic Systems, vol. 87, no. 3-4, pp. 615-626, 2017.

[25] K. T. Fang and C. X. Ma. Design method, Design Method of Orthogonal And Uniform Test, Science Press, Beijing, China, 2001.

[26] V. K. Lakshminarayan and J. D. Baeder, "Computational investigation of micro hovering rotor aerodynamics," Journal of the American Helicopter Society, vol. 55, no. 2, pp. 022001102200125, 2010. 


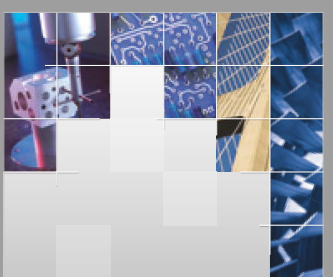

\section{Enfincering}
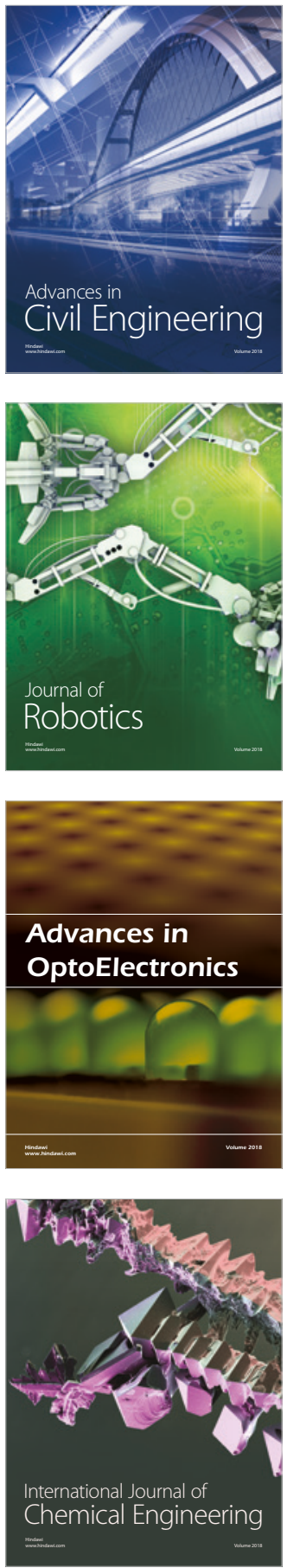

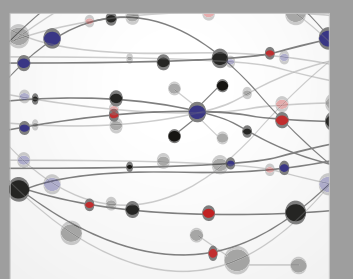

\section{Rotating \\ Machinery}

The Scientific World Journal

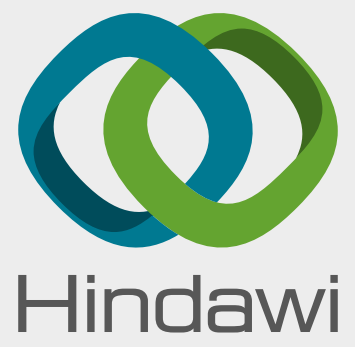

Submit your manuscripts at

www.hindawi.com
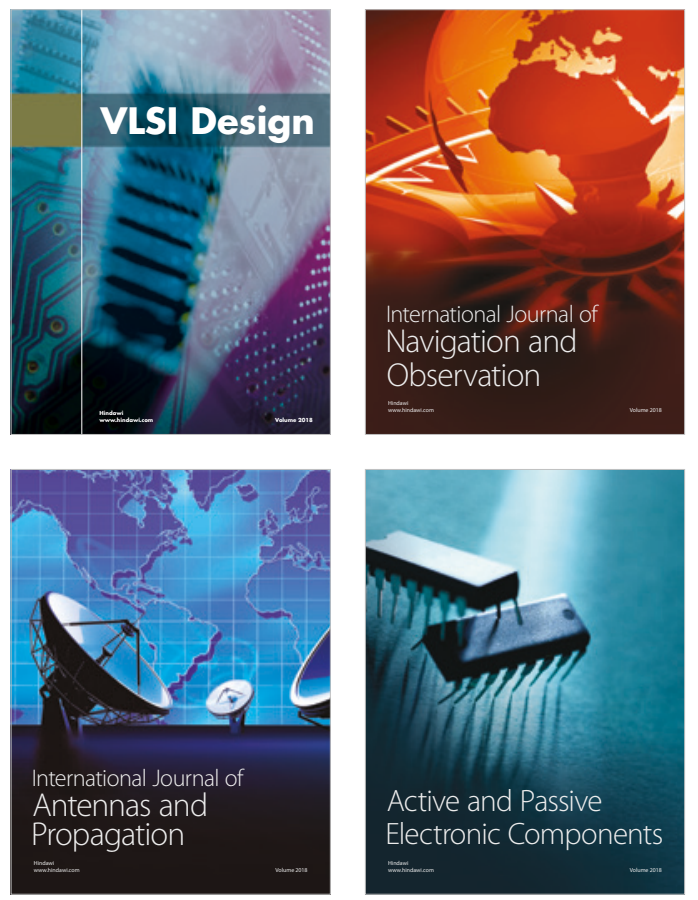
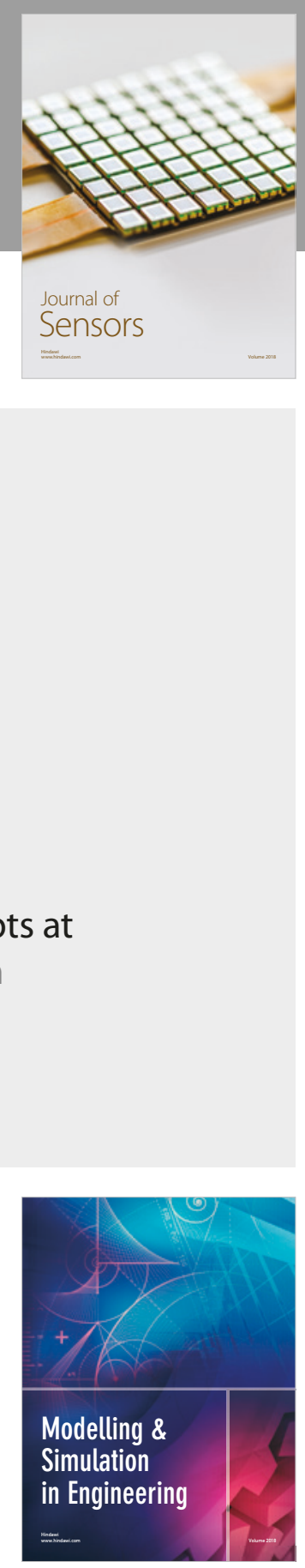

\section{Advances \\ Multimedia}
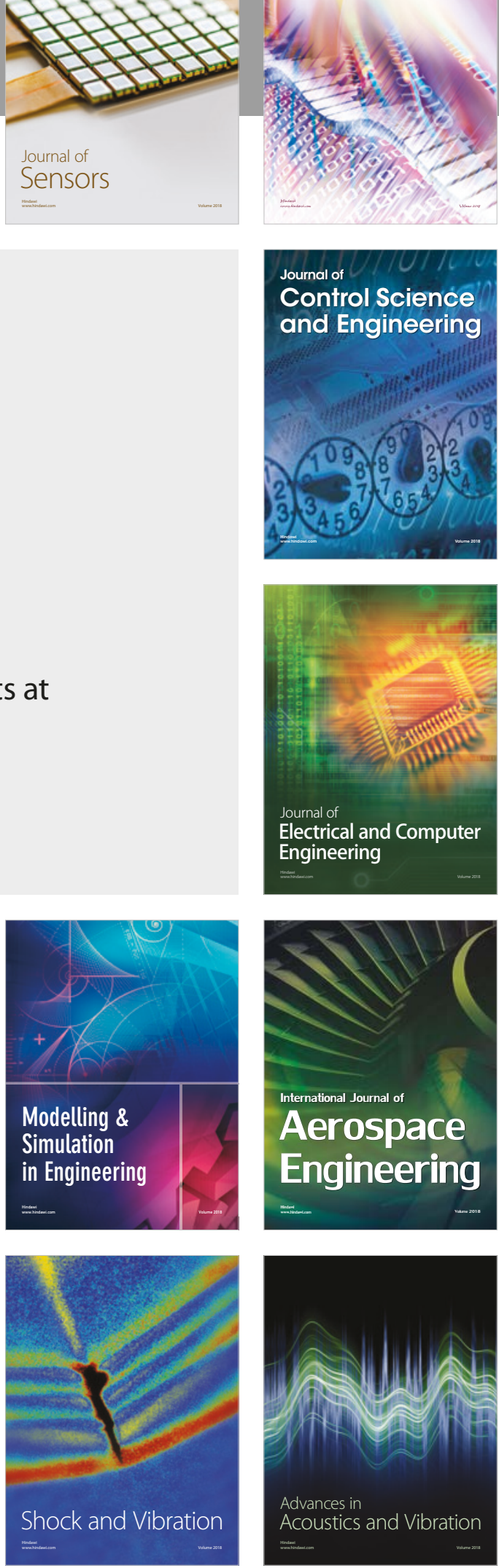